\title{
Novel interpretation strategy for searches of singly produced vectorlike quarks at the LHC
}

\author{
Avik Roy $\odot,{ }^{1, *}$ Nikiforos Nikiforou $\odot,{ }^{1, \dagger}$ Nuno Castro $\oplus^{2,3, *}$ and Timothy Andeen $\circledast^{1, \S}$ \\ ${ }^{1}$ Center for Particles and Fields, Department of Physics, \\ University of Texas at Austin, Austin, Texas 78712, USA \\ ${ }^{2}$ Laboratório de Instrumentação e Física Experimental de Partículas (LIP), \\ Universidade do Minho, 4710-057 Braga, Portugal \\ ${ }^{3}$ Departamento de Física, Escola de Ciências, Universidade do Minho, 4710-057 Braga, Portugal
}

(Received 6 March 2020; accepted 1 June 2020; published 22 June 2020)

\begin{abstract}
Vectorlike quarks (VLQs) are potential signatures of physics beyond the standard model at the TeV energy scale, and major effort has been put forward at both the ATLAS and CMS experiments to search for these particles. To make these search results more relatable in the context of most plausible theories of VLQs, it is deemed important to present the analysis results in a general fashion. We investigate the challenges associated with such interpretations of singly produced VLQ searches and propose a generalized, semianalytical framework that allows for a model-independent casting of the results in terms of unconstrained free parameters of the VLQ Lagrangian. We also propose a simple parametrization of the correction factor to the single VLQ production cross section at large decay widths. We illustrate how the proposed framework can be used to conveniently represent statistical limits by numerically reinterpreting results from benchmark ATLAS and CMS analyses.
\end{abstract}

DOI: $10.1103 /$ PhysRevD.101.115027

\section{INTRODUCTION}

Vectorlike quarks (VLQs), whose existence was predicted by a number of beyond the standard model theories [1-10] at the TeV scale, are excellent search candidates in the post-Higgs era at CERN's Large Hadron Collider (LHC). VLQs are $S U(3)$ color triplets that have the same strong coupling as the standard model quarks but maintain identical electroweak representations for both chiralities. The spectrum of the VLQ species consists of four particles, denoted as $X_{+\frac{5}{3}}, T_{+\frac{2}{3}}, B_{-\frac{1}{3}}$, and $Y_{-\frac{4}{3}}$, where the subscript indicates the electric charge of the corresponding particle. They can exist as $(T)$ or $(B)$ singlets, $(X, T),(T, B)$, or $(B, Y)$ doublets, and $(X, T, B)$ or $(T, B, Y)$ triplets. In most representations, they couple to the standard model quarks via an exchange of charged $\left(W^{+}, W^{-}\right)$or neutral $(Z, H)$ bosons.

\footnotetext{
*aroy@utexas.edu

nikiforos.nikiforou@cern.ch

*nuno.castro@cern.ch

\$tandeen@utexas.edu
}

Published by the American Physical Society under the terms of the Creative Commons Attribution 4.0 International license. Further distribution of this work must maintain attribution to the author(s) and the published article's title, journal citation, and DOI. Funded by SCOAP.
The search efforts for VLQs in collider experiments such as ATLAS and CMS can be broadly categorized into two classes: (a) seaches for VLQ pairs and (b) searches for singly produced VLQs. In general, each analysis targets a generic final state that is dominantly sensitive to one, or occasionally more than one, decay mode of the VLQs. Searches for pair production of VLQs have been traditionally more popular than searches for singly produced VLQs. This is primarily because, in many theoretical models, pair production of VLQs is dominated by a model-independent, strong-force-mediated process [Fig. 1(a)]. However, it should be noted that alternate production modes of pair produced VLQs, e.g., via heavy gluons [11,12], and the interpretation of VLQ search results in the context of such models [13] have also been explored. Using the data

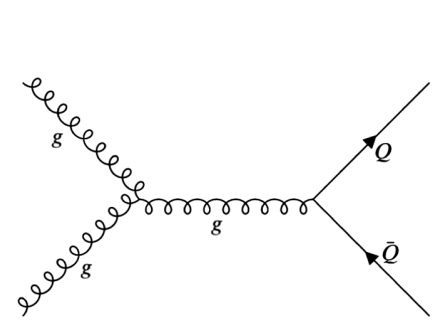

(a)

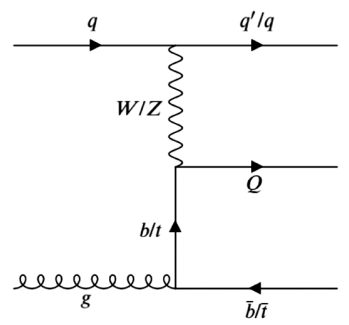

(b)
FIG. 1. Dominant contributing diagrams for (a) pair production and (b) single production of VLQs. 
collected at $8 \mathrm{TeV}$ center of mass energy during Run 1 at the LHC between 2009 and 2013, a number of analyses concentrated on pair production of VLQs [14-19]. Although no significant excess was seen in the data, each analysis independently sets exclusion limits on a VLQ mass in the range of approximately $600-1000 \mathrm{GeV}$ that has served as the benchmark for the complementary Run 2 searches. Similar limits were obtained from the searches that focused on single production of VLQs in Run $1[16,20]$.

At a center of mass energy of $13 \mathrm{TeV}$ in Run 2, the ATLAS Collaboration has not only performed a number of searches looking for the pair production of VLQs [21-25] but also combined the results of these analyses to set the strongest current limits on the VLQ masses [26]. Complementary pair production analyses from CMS [27-29] have also set limits in the range of $\mathcal{O}(1 \mathrm{TeV})$ for up- and down-type VLQs.

During Run 2, there has been a significant increase in the number of searches of singly produced VLQs [30-36]. This is partly because, depending on how strongly VLQs couple with standard model (SM) bosons and quarks, single production processes can have a larger cross section at the range of masses that Run 2 searches have been focusing on [37]. However, unlike pair production, the production of single VLQs is dominated by electroweak processes [Fig. 1(b)], and they decay via an exchange of electroweak gauge bosons and the Higgs boson. Hence, production and decay of single VLQs depend on the electroweak representation of these heavy fermions. Different analyses have adopted different strategies, often inspired by model-specific assumptions. Results obtained with these analyses cannot be consistently compared or combined because of the diverse set of assumptions and model-dependent interpretation strategies.

This paper aims to lay out an experimentally inspired, semianalytical framework for a relatively model-independent interpretation of single VLQ production search results that can be adopted by most ongoing and future analyses. In Sec. II, we explain the details and challenges of a modelindependent interpretation of single VLQ production searches and emphasize why such a strategy is important. Section III introduces a minimal set of assumptions and presents the semianalytical framework. In Sec. IV, we introduce a novel parametrization for estimating the correction to the single VLQ production cross section at finite widths. Finally, in Sec. V, we demonstrate how this framework can be used to compare, reinterpret, and visualize existing search results from ATLAS and CMS.

\section{THE PHILOSOPHY OF INTERPRETATION OF VLQ SEARCHES}

A standard experimental search for VLQs benefits from a relatively model-independent parametrization of these particles. Such a representation utilizes a collection of arbitrary parameters - the VLQ masses $\left\{M_{Q}\right\}$ and their couplings $\vec{c}=\left\{c_{L / R, V / H}^{Q q}\right\}$ to the standard model quarks via an exchange of the gauge bosons $V \in\left\{W^{ \pm}, Z\right\}$ and the Higgs boson $H$. An experimental search evaluates the statistically excluded cross section, $\sigma_{\lim }\left(M_{Q}, \vec{c}\right)$, for a grid of points of the parametric hyperspace. These limits can be interpreted in the context of a certain theoretical model as long as the model does not dramatically deviate from the assumptions of the model-independent representation. This approach has been dubbed the bridge model by Matsedonskyi et al. [38]. In the same paper, the authors describe the following simplified Lagrangian for VLQs in terms of these generalized couplings,

$\mathcal{L}=\sum_{\zeta, q, Q}\left[\frac{g_{w}}{2} \sum_{V} c_{\zeta, V}^{Q q} \bar{Q}_{\zeta} \forall q_{\zeta}+c_{\zeta, H}^{Q q} H \bar{Q}_{\zeta^{\prime}} q_{\zeta}\right]+$ H.c.,

where $Q$ represents the usual VLQs $\left\{X_{+\frac{5}{3}}, T_{+\frac{2}{3}}, B_{-\frac{1}{3}}, Y_{-\frac{4}{3}}\right\}$, $\zeta$ and $\zeta^{\prime}$ represent alternate chiralities, and $q$ represents a SM quark of up or down type. Some of these couplings may be constrained by the conservation of certain quantum numbers. For example, the $+\frac{5}{3}$ charged partner $X$ can couple with SM up-type quarks only with the exchange of a $W$ boson. The parametic hyperspace of this modelindependent representation can be mapped to those of similar formulations in [37,39-42] by a one-to-one correspondence among the tree-level couplings.

Pair production of VLQs has inspired an elegant interpretation strategy. The dominant QCD-facilitated production mode for a pair of VLQs allows a model-independent estimate of the cross section for the pair production under a narrow width approximation (NWA), i.e.,

$$
\sigma\left(p p \rightarrow Q \bar{Q} \rightarrow V_{1} q_{1} V_{2} \bar{q}_{2} ; M_{Q}, \vec{c}\right) \stackrel{\mathrm{NWA}}{\longrightarrow} \sigma_{\mathrm{prod}, Q \bar{Q}}^{\mathrm{NW}}\left(M_{Q}\right) \times \mathrm{BR}\left(Q \rightarrow V_{1} q_{1} ; \vec{c}\right) \times \mathrm{BR}\left(Q \rightarrow V_{2} q_{2} ; \vec{c}\right)
$$

Here, the value of the production cross section $\sigma_{\text {prod, } Q \bar{Q}}^{\mathrm{NW}}\left(M_{Q}\right)$ is independent of the electroweak group representation and the corresponding coupling parameters of the heavy fermions. This allows for a reformulation of the VLQ Lagrangian by treating the branching ratios themselves as free parameters while ignoring their complex dependence on the coupling parameters $\vec{c}$. Moreover, most analyses looking for these heavy fermionic resonances also assume that these particles couple predominantly only with the third generation of standard model quarks, i.e., 
$\mathrm{BR}(Q \rightarrow H q)+\mathrm{BR}(Q \rightarrow Z q)+\mathrm{BR}\left(Q \rightarrow W q^{\prime}\right)=1.0$,

where $q, q^{\prime} \in\{t, b\}$.

The assumption on the branching ratios in Eq. (3) allows for, along with the NWA, a simple interpretation for searches of pair production of VLQs, where the excluded region in the parametric hyperspace is evaluated by solving the following inequality:

$$
\sigma_{\operatorname{prod}, Q \bar{Q}}^{\mathrm{NW}}\left(M_{Q}\right) \geq \sigma_{\lim , Q \bar{Q}}^{\mathrm{NW}}\left(M_{Q}, \mathrm{BR}_{W}, \mathrm{BR}_{H}\right),
$$

where $\sigma_{\lim , Q \bar{Q}}^{\mathrm{NW}}$ is the statistically excluded cross-section limit (usually computed at $95 \%$ confidence level) at narrow width, corresponding to the largest process cross section compatible with the background only hypothesis given the observed distribution in data. The cross-section limit depends on the sensitivity of an analysis to different VLQ decay modes and hence is a function of the choice of VLQ decay branching fractions. As a result, a parametrically model-independent interpretation of pair production searches can be done by solving Eq. (4) for some chosen grid of allowed values of $\operatorname{BR}\left(Q \rightarrow W q^{\prime}\right)$, $\operatorname{BR}(Q \rightarrow H q)$ to set a limit on the VLQ mass.

The importance of a universal, model-independent interpretation strategy is pivotal for a combination of multiple analyses. A consistent combination of multiple analyses requires a well-defined correlation scheme among the various nuisance parameters as well as the parameter(s) of interest [POI(s)], with the latter usually being a function of the signal cross section. A generalized interpretation strategy, as given in Eq. (4), can be used to formulate a well-defined correlation scheme for the POIs of different analyses. Different analyses tend to be sensitive to different

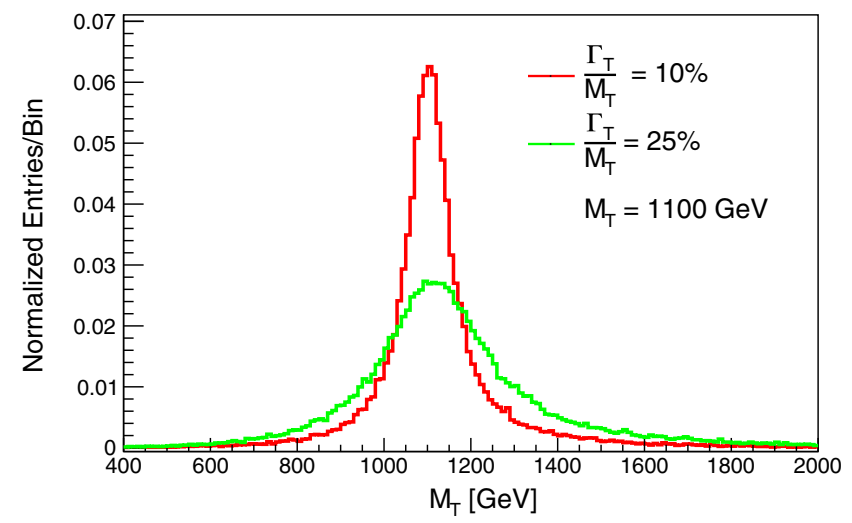

(a) kinematic signatures and different regions of phase space. A combination of such analyses guided by a well-defined interpretation strategy can significantly boost the statistical power and hence set stronger limits on the parametric hyperspace. The combination [26] performed by the ATLAS Collaboration excluded up- (down-) type VLQ masses up to 1.31 (1.03) $\mathrm{TeV}$ for any combination of branching ratios with respect to Eq. (3).

In light of the interpretation strategy for pair produced VLQs given in Eq. (4), a framework of interpreting search results for singly produced VLQs can be laid out. The excluded region of parametric hyperspace can be evaluated by solving the inequality

$$
\sigma_{V Q A q}\left(M_{Q}, \vec{c}\right) \geq \sigma_{\lim , V Q A q}\left(M_{Q}, \vec{c}\right),
$$

where $V Q A q$ is shorthand notation for the production of the VLQ $Q$ being mediated by the vector boson $V$ that subsequently decays to the boson $A$ and SM quark $q$. The production and decay of single VLQs involve the relevant couplings at the corresponding vertices. The same couplings determine the partial decay widths and hence the branching ratios of these VLQs in the associated decay channels. Moreover, the kinematic distributions of VLQ decay products also change with the change of VLQ decay widths and hence with the choice of couplings (Fig. 2). This changes the phase space sensitivity of the analyses and, as a result, the exclusion limit also becomes nontrivially dependent on the choice of not only the VLQ mass but also the couplings.

The nontrivial coupling dependence of the interpretation relation in Eq. (5) makes it somewhat challenging to formulate a generalized interpretation strategy. Previous ATLAS and CMS analyses incorporated simplified interpretation strategies by making model-dependent assumptions

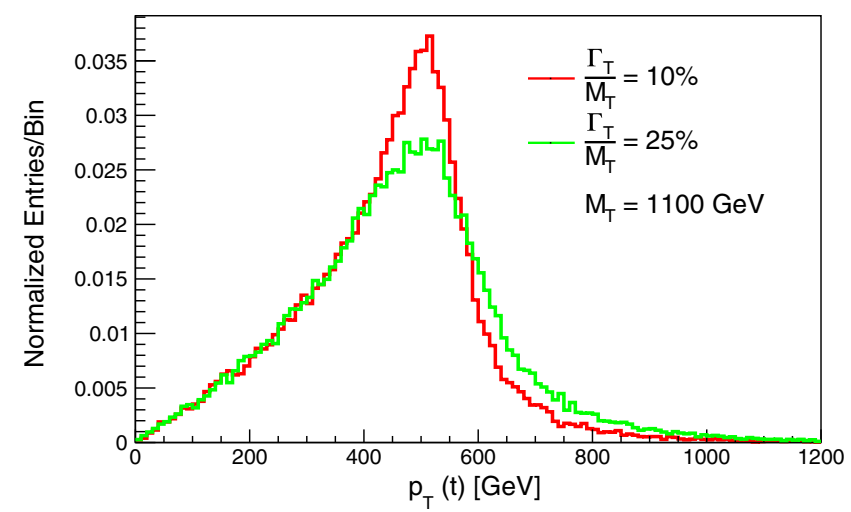

(b)

FIG. 2. Distribution of (a) VLQ invariant mass and (b) transverse momentum of the top quark from its decay in association with a $Z$ boson for a $+\frac{2}{3}$ charged top partner $(T)$. These distributions were obtained from a Monte Carlo simulation of singly produced $T$ using MadGraph_amC@NLO $[43,44]$. The coupling values are set to $c_{L, W}=0.5$ (red lines) and $c_{L, W}=0.8$ (green lines) with branching ratios assumed to be $50 \%, 25 \%$, and $25 \%$ for the $\mathrm{Wb}, \mathrm{Zt}$, and $\mathrm{Ht}$ decay modes, respectively, to obtain the desired relative decay width. All histograms are normalized to unity. 
about either the branching ratios [20,31] or the relative decay widths $[33,34]$, or by interpreting the generic couplings in terms of the mixing angles between the heavy fermions and their SM counterparts [30-32]. Such a diversity of interpretation strategies often makes it difficult to compare results from different analyses and formulate a consistent correlation scheme for a possible combination of such analyses.

In Sec. III, we present a formulation of a generalized interpretation strategy for singly produced VLQs. Following in the footsteps of the interpretation strategy of searches for the pair production of VLQs, we pursue a general idea of making a set of strategic assumptions to reduce the dimensions of the parametric hyperspace providing the avenue of translating the exclusion limits to excluded regions of the reduced hyperspace.

\section{FRAMEWORK FOR INTERPRETATION OF SINGLY PRODUCED VLQS}

The first assumption that we make is to restrict VLQs to interact with third generation SM quarks exclusively. Additionally, we exclude models that incorporate the single production of VLQs in association with heavy vector bosons [45,46] or their decay via exotic scalars [47-52], although such models are often theoretically well motivated. This results in the branching fractions being constrained according to Eq. (3). In light of this assumption, we will drop the superscript indices $Q q$ in the generalized couplings from this point forward - this association will be clear from the context of the discussion.

Secondly, we assume that VLQs are much heavier than SM fermions and bosons, i.e.,

$$
M_{Q} \gg m_{t},
$$

where $m_{t}$ denotes the top quark mass of $172.5 \mathrm{GeV}$ [53]. This assumption was inspired by the model-independent pair production search results from the ATLAS and CMS collaborations, which set a limit on VLQ masses in the range of $\mathcal{O}(1 \mathrm{TeV})$ independently of its electroweak representation. At the large $M_{Q}$ limit, the interference term between left- and right-handed couplings in the analytic expressions of the partial decay widths becomes negligible. Therefore, the corresponding partial decay widths for $Q \rightarrow V q$ and $Q \rightarrow H q$ can be approximated as

$$
\begin{gathered}
\Gamma(Q \rightarrow V q) \approx\left(c_{L, V}^{2}+c_{R, V}^{2}\right) \times \frac{g_{w}^{2}}{32 \pi} \frac{p\left(M_{Q}, m_{q}, m_{V}\right)}{M_{Q}^{2}}\left(\frac{M_{Q}^{2}+m_{q}^{2}}{2}+\frac{\left(M_{Q}^{2}-m_{q}^{2}\right)^{2}}{2 m_{V}^{2}}-m_{V}^{2}\right), \\
\Gamma(Q \rightarrow H q) \approx\left(c_{L, H}^{2}+c_{R, H}^{2}\right) \times \frac{1}{8 \pi} \frac{p\left(M_{Q}, m_{q}, m_{H}\right)}{M_{Q}^{2}} \frac{M_{Q}^{2}+m_{q}^{2}-m_{H}^{2}}{2},
\end{gathered}
$$

where $g_{w}$ represents the electroweak coupling constant and

$$
p(X, y, z)=\frac{1}{2 X} \sqrt{\left[X^{2}-(y+z)^{2}\right]\left[X^{2}-(y-z)^{2}\right]} .
$$

Since the decay width expressions depend only on the quadrature sum of the left- and right-handed couplings, we introduce a more convenient set of notations:

$$
c_{W / Z / H}^{2}=c_{L, W / Z / H}^{2}+c_{R, W / Z / H}^{2} .
$$

Defining $r_{A}=\frac{m_{A}}{M_{Q}}$, we introduce the following functions:

$$
\begin{aligned}
\rho_{W}(Q) & =\sqrt{1+r_{W}^{4}+r_{q}^{4}-2 r_{W}^{2}-2 r_{q}^{2}-2 r_{W}^{2} r_{q}^{2}}\left(1+r_{W}^{2}-2 r_{q}^{2}-2 r_{W}^{4}+r_{q}^{4}+r_{W}^{2} r_{q}^{2}\right), \\
\rho_{Z}(Q) & =\sqrt{1+r_{Z}^{4}+r_{q}^{4}-2 r_{Z}^{2}-2 r_{q}^{2}-2 r_{Z}^{2} r_{q}^{2}}\left(1+r_{Z}^{2}-2 r_{q}^{2}-2 r_{Z}^{4}+r_{q}^{4}+r_{Z}^{2} r_{q}^{2}\right), \\
\rho_{H}(Q) & =\sqrt{1+r_{H}^{4}+r_{q}^{4}-2 r_{H}^{2}-2 r_{q}^{2}-2 r_{H}^{2} r_{q}^{2}}\left(1+r_{q}^{2}-r_{H}^{2}\right) .
\end{aligned}
$$

These functions evaluate to unity at leading order, i.e., $\rho_{W} \approx \rho_{Z} \approx \rho_{H} \approx 1.0$, because $r \ll 1$. They introduce some minor mass-dependent corrections that vanish for large values of $M_{Q}$. To simplify the expression of the branching ratios, we rescale the coupling parameters as follows: 


$$
\begin{aligned}
& c_{W}^{2}=c_{L, W}^{2}+c_{R, W}^{2}=\tilde{c}_{W}^{2}, \\
& c_{Z}^{2}=c_{L, Z}^{2}+c_{R, Z}^{2}=\tilde{c}_{Z}^{2} \frac{m_{Z}^{2}}{m_{W}^{2}}, \\
& c_{H}^{2}=c_{L, H}^{2}+c_{R, H}^{2}=\frac{g_{w}^{2}}{4} \tilde{c}_{H}^{2} \frac{M_{Q}^{2}}{m_{W}^{2}} .
\end{aligned}
$$

Expressed in terms of the rescaled couplings and the $\rho_{A}(Q)$ functions, the decay widths become

$$
\Gamma(Q \rightarrow A q)=\tilde{c}_{A}^{2} \times \frac{g_{w}^{2}}{128 \pi} \frac{M_{Q}^{3}}{m_{W}^{2}} \times \rho_{A}(Q) .
$$

Then the equation for the branching ratio reduces to

$$
\operatorname{BR}(Q \rightarrow A q)=\frac{\tilde{c}_{A}^{2} \rho_{A}(Q)}{\tilde{c}_{W}^{2} \rho_{W}(Q)+\tilde{c}_{Z}^{2} \rho_{Z}(Q)+\tilde{c}_{H}^{2} \rho_{H}(Q)} .
$$

Hence, the assumption in Eq. (6) allows the branching fractions to be independent of the chirality of the couplings. The same assumption makes the production cross section of single VLQs independent of the choice of the chirality at leading order. For example, as argued by Matsedonskyi et al. [38], the production cross section for $Z$ - and $W$-boson-mediated production modes under the narrow width approximation is approximated as

$$
\begin{aligned}
\sigma_{\text {prod }, V Q}^{\mathrm{NW}}\left(M_{Q}, \vec{c}\right) \approx & \left(c_{V}^{2}+k \times c_{L, V} c_{R, V} \frac{m_{q}}{m_{q}+M_{Q}}\right) \\
& \times \sigma_{\text {prod }, V Q}^{\mathrm{NW}}\left(M_{Q}, c_{V}=1\right),
\end{aligned}
$$

where $k$ is a constant of $\mathcal{O}(1)$. Assuming that $M_{Q} \gg m_{q}$, as in Eq. (6), suppresses the interfering term by $\mathcal{O}\left(\frac{m_{q}}{M_{Q}}\right)$. Furthermore, Aguilar-Saavedra et al. proved in [37] that, independent of the representation, one of the chiral couplings is suppressed by an additional factor of $\mathcal{O}\left(\frac{m_{q}}{M_{Q}}\right)$ that emerges from the diagonalization of the mass matrix. As a result, the contribution of the interference term in Eq. (14) will be of subleading order and can be ignored. Based on similar arguments, it was also assumed in [39] that one of the chiralities dominates the model-independent representation of the VLQs. Many analyses, in both the ATLAS and CMS collaborations, have also reported that their results are independent of the chiral structure of the couplings - the effect of different chiralities is indistinguishable within a coarse binning structure in the discriminant variables as well as the statistical and systematic uncertainties that dominate the limit setting $[30,32,33,35,36]$. Hence, we introduce our third assumption: either an analysis is insensitive to the relative structure of the chiral couplings or a single chirality dominates the signal kinematics. As a result, the statistical limits obtained from an analysis, $\sigma_{\text {lim }}$, now depend on $M_{Q}$ and the chirality-ignorant rescaled couplings in Eq. (11), i.e., $\vec{c}=\left\{\tilde{c}_{W}, \tilde{c}_{Z}, \tilde{c}_{H}\right\}$.

The three assumptions made so far can be summarized as follows:

(a) VLQs as top or bottom partners.-VLQs predominantly couple to third generation SM quarks via an exchange of $W, Z$, and $H$ bosons.

(b) Heavy VLQs.-VLQs are much heavier than SM bosons and fermions.

(c) Chirality-agnostic analysis.-The analysis of the search for a single VLQ is either insensitive to the relative chiral structure or dominated by a single chirality of the couplings.

Given the aforementioned set of assumptions, we can now derive the explicit expression for the inequality in Eq. (5). We can write the $V Q A q$ process cross section as a product of the production cross section and the corresponding branching ratio for narrow widths:

$$
\begin{aligned}
\sigma_{V Q A q}\left(M_{Q}, \vec{c}\right) & \stackrel{\mathrm{NWA}}{\longrightarrow} \sigma_{\mathrm{prod}, V Q}^{\mathrm{NW}}\left(M_{Q}, \vec{c}\right) \times \mathrm{BR}(Q \rightarrow A q) \\
& =\tilde{c}_{V}^{2} \times \sigma_{\mathrm{prod}, V Q}^{\mathrm{NW}}\left(M_{Q}, \tilde{c}_{V}=1\right) \times \mathrm{BR}(Q \rightarrow A q),
\end{aligned}
$$

where the branching ratio is given by Eq. (13). For larger widths, this estimate for the cross section is corrected for the width dependence of the process cross section. Following the recipe of [30,31], we define the correction factor as

$$
\mathrm{P}_{\mathrm{NWA}}\left(M_{Q}, \vec{c}\right)=\frac{\sigma_{\mathrm{prod}, V Q}^{\mathrm{NW}} \times \mathrm{BR}(Q \rightarrow A q)}{\sigma_{V Q A q}} .
$$

Together with Eqs. (15) and (16), the interpretation relation in Eq. (5) reduces to

$$
\frac{\tilde{c}_{V}^{2} \times \sigma_{\mathrm{prod}, V Q}^{\mathrm{NW}}\left(M_{Q}, \tilde{c}_{V}=1\right) \times \mathrm{BR}(Q \rightarrow A q)}{\mathrm{P}_{\mathrm{NWA}}\left(M_{Q}, \vec{c}\right)} \geq \sigma_{\lim , V Q A q}\left(M_{Q}, \vec{c}\right) .
$$


TABLE I. The choice of couplings for event generation and cross-section calculation in MadGraph_aMC@NLO.

\begin{tabular}{ll}
\hline \hline Process & Coupling choice \\
\hline$W T W b, W T Z t, Z T W b$, & $\tilde{c}_{W}, \tilde{c}_{Z} \in C$ \\
$W B W t, W B Z b, Z B W t$ & $\tilde{c}_{H}=0$ or $\tilde{c}_{H}=\tilde{c}_{Z}$ \\
$W T H t$, & $\tilde{c}_{W}, \tilde{c}_{H} \in C$ \\
$W B H b$ & $\tilde{c}_{Z}=0$ or $\tilde{c}_{Z}=\tilde{c}_{H}$ \\
$Z T Z t, Z T H t$, & $\tilde{c}_{Z}, \tilde{c}_{H} \in C$ \\
$Z B Z b, Z B H b$ & $\tilde{c}_{W}=0$ or $\tilde{c}_{W}=\tilde{c}_{H}$ \\
\hline \hline
\end{tabular}

\section{EVALUATION OF $\mathbf{P}_{\mathrm{NWA}}$}

In the limit of narrow decay width, the Breit-Wigner distribution can be approximated as a delta function:

$$
\frac{1}{\left(p^{2}-M^{2}\right)^{2}+\Gamma^{2} M^{2}} \stackrel{\stackrel{\Gamma}{M} \rightarrow 0}{\longrightarrow} \frac{\pi}{M \Gamma} \delta\left(p^{2}-M^{2}\right)
$$

For large widths, this approximation breaks down and the cross-section estimate accumulates corrections in the higher order of $\frac{\Gamma}{M}[54,55]$. It is reasonable to assume that the correction factor for the cross section will depend only on the values of $\Gamma_{Q}$ and $M_{Q}$ and not on the individual choices for the couplings. As a result, the analytic expression for the correction factor takes the following form:
$\mathrm{P}_{\mathrm{NWA}}\left(M_{Q}, \vec{c}\right) \equiv \mathrm{P}_{\mathrm{NWA}}\left(\frac{\Gamma_{Q}}{M_{Q}}\right) \approx 1+\sum_{n} A_{n}\left(\frac{\Gamma_{Q}}{M_{Q}}\right)^{n}$,

where the values $A_{n}$ will depend on the choice of the VLQ mass and the process of interest.

The traditional parametrization of the VLQ Lagrangian, as given in Eq. (1) and its equivalent formulations, allow signal event generation by fixing the coupling parameters, while the partial decay widths can be calculated from Eq. (12). Therefore, using the VLQ Universal FeynRules Output model, inspired by the parametrization presented in [40], single production of top and bottom partners for both $W$ - and $Z$-boson-mediated single- $T$ and single- $B$ processes for $M_{Q}$ in the range $1000-2200 \mathrm{GeV}$ in steps of $200 \mathrm{GeV}$ was simulated in MadGraph_aMC@NLO. The actual choice of coupling values in relation to the representative set of couplings $C=$ $\{0.05,0.2,0.4,0.6,0.8,1.0\}$ is summarized in Table I. We additionally require that $\frac{\Gamma_{Q}}{M_{Q}}<0.5$ for any choice of couplings and disregard any coupling combination that violates this constraint. The $\kappa, \hat{\kappa}, \tilde{\kappa}$ parameters introduced in [40] can be calculated from the rescaled couplings from a one-to-one correspondence with the tree-level couplings in Eq. (1).

We calculated the narrow width and large width cross section at leading order for all of the processes mentioned in Table I. Using Eq. (16), we evaluated the correction factor for each choice of coupling and mass.

Figures 3 and 4, respectively, show the variation in $\mathrm{P}_{\mathrm{NWA}}$ as a function of $\frac{\Gamma}{M}$ for the WTZt and WTHt processes.

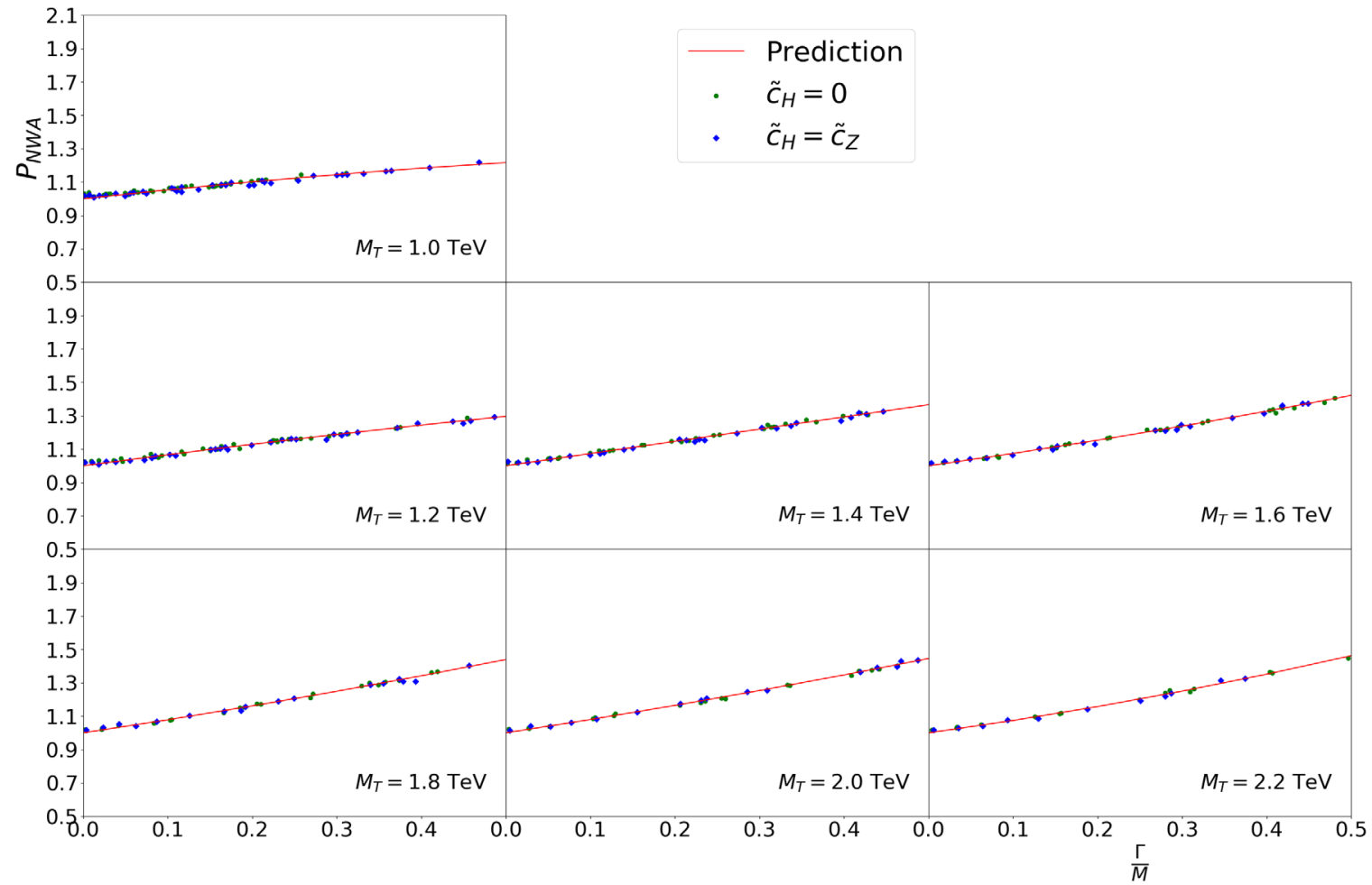

FIG. 3. Estimated values of $\mathrm{P}_{\mathrm{NWA}}$ plotted as a function of $\frac{\Gamma}{M}$ for different values of $M_{T}$ for the $W T Z t$ process. The red line shows the best fit estimate for the correction factor according to the quadratic polynomial in Eq. (20). 


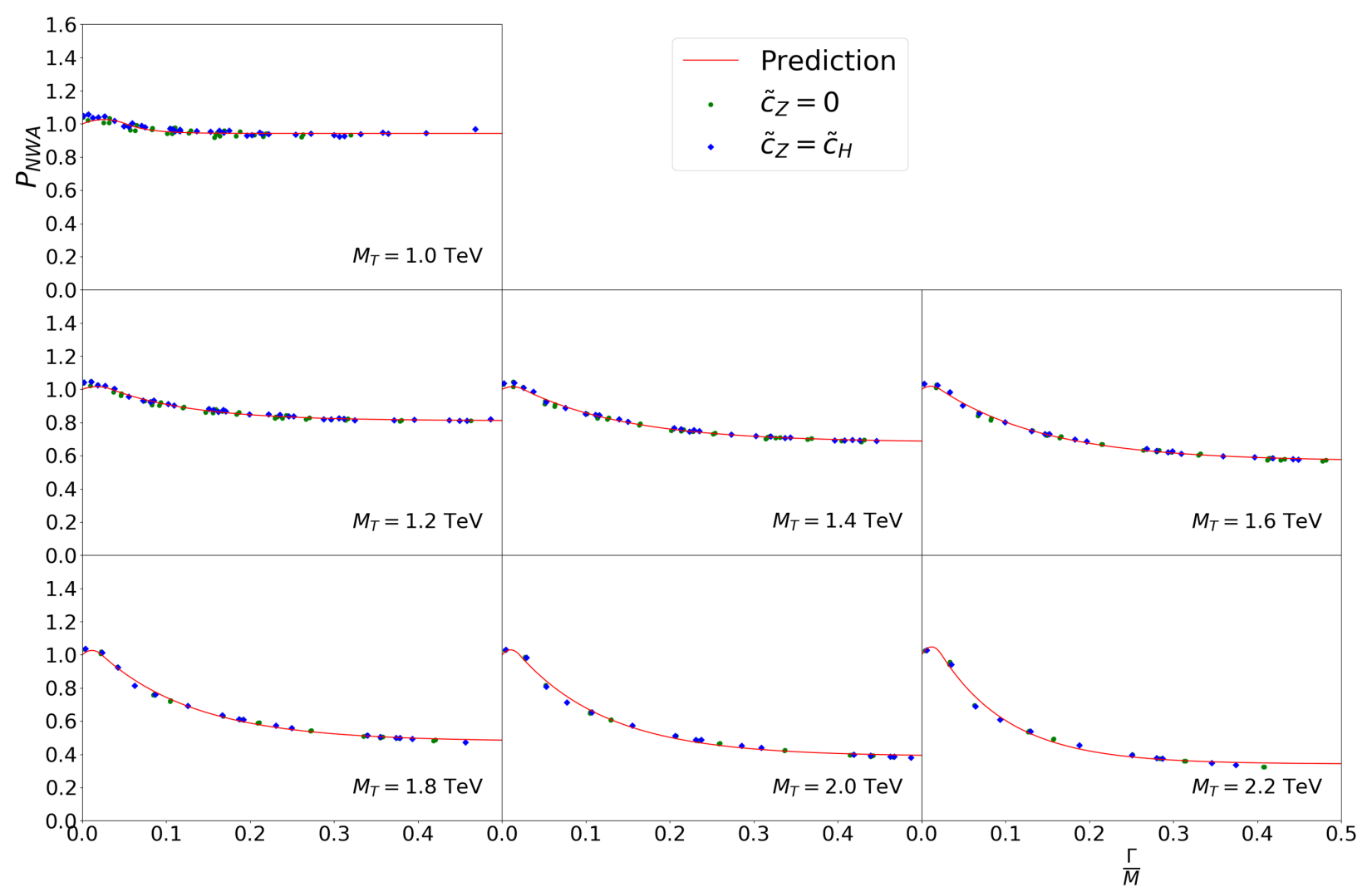

FIG. 4. Estimated values of $\mathrm{P}_{\mathrm{NWA}}$ plotted as a function of $\frac{\Gamma}{M}$ for different values of $M_{T}$ for the WTHt process. The red line shows the best fit estimate according to the functional form in Eq. (21).

Confirming the initial assumption, there is no strong dependence on the choice of couplings. For example, as can be seen in Fig. 3, for a given value of $\frac{\Gamma_{Q}}{M_{Q}}$, the correction factor calculated for $\tilde{c}_{H}=0$ is almost identical to the one calculated for $\tilde{c}_{H}=\tilde{c}_{Z}$.

We also observe in Fig. 3 that $\mathrm{P}_{\mathrm{NWA}}$ monotonically rises from unity in the case of the $W T Z t$ processes. The differential cross-section distributions for $W T Z t$ processes of a $1.6 \mathrm{TeV}$ top partner for different choices of the VLQ couplings are shown in Fig. 5. Accounting for increased width causes a decrease in total cross section because, as the VLQ kinematics reach a phase space away from the pole mass, the matrix element receives a compensating contribution from the VLQ propagator. The functional behavior of $\mathrm{P}_{\mathrm{NWA}}$ is well approximated by a quadratic polynomial for processes that incorporate a decay to the vector bosons:

$$
\mathrm{P}_{\mathrm{NWA}, V Q V q}\left(\frac{\Gamma_{Q}}{M_{Q}}\right)=1+A_{1} \frac{\Gamma_{Q}}{M_{Q}}+A_{2}\left(\frac{\Gamma_{Q}}{M_{Q}}\right)^{2}
$$

The $A_{1}$ and $A_{2}$ parameters in Eq. (20) for different processes with top and bottom partners decaying to vector bosons are evaluated by obtaining the least squared error fit to the observed values of the correction factor from simulation. They are tabulated in Tables II and III, respectively.

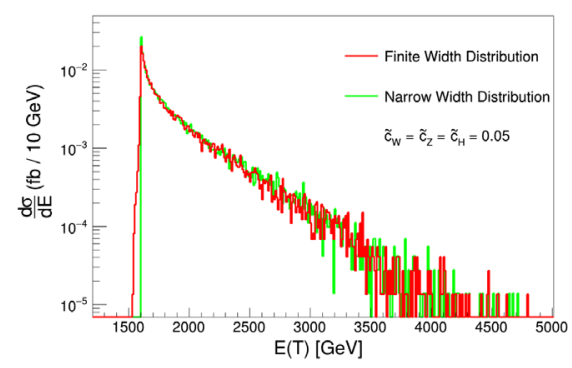

(a)

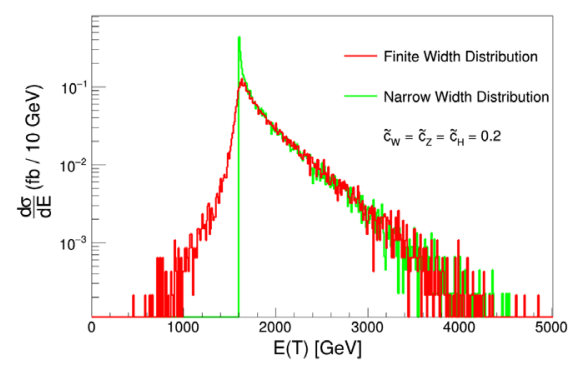

(b)

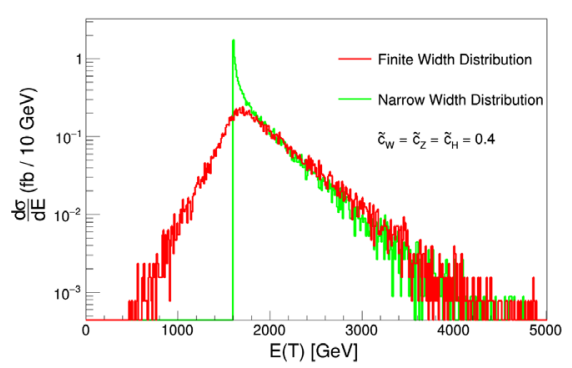

(c)

FIG. 5. Distribution of a differential cross section of singly produced top partner $(T)$ of mass $M_{T}=1.6$ TeV at narrow width and finite width as obtained from MadGraph_amC@NLO simulation of WTZt process. The coupling values $\tilde{c}_{W}$ are set at 0.05 , 0.20, and 0.40, respectively, in (a)-(c). All distributions assume that $\tilde{c}_{W}=\tilde{c}_{Z}=\tilde{c}_{H}$. 
TABLE II. The best fit values for the parametric representation of $\mathrm{P}_{\mathrm{NWA}}$ in Eq. (20) for different values of $M_{T}$.

\begin{tabular}{|c|c|c|c|c|c|c|c|c|}
\hline \multirow[b]{2}{*}{$M_{T}(\mathrm{TeV})$} & \multicolumn{2}{|c|}{$W T W b$} & \multicolumn{2}{|c|}{$W T Z t$} & \multicolumn{2}{|c|}{$Z T W b$} & \multicolumn{2}{|c|}{$Z T Z t$} \\
\hline & $A_{1}$ & $A_{2}$ & $A_{1}$ & $A_{2}$ & $A_{1}$ & $A_{2}$ & $A_{1}$ & $A_{2}$ \\
\hline 1.0 & 0.526 & -0.123 & 0.557 & -0.247 & 0.507 & -0.272 & 0.374 & 0.013 \\
\hline 1.2 & 0.638 & -0.048 & 0.681 & -0.176 & 0.639 & -0.126 & 0.550 & 0.035 \\
\hline 1.4 & 0.708 & 0.054 & 0.733 & -0.001 & 0.737 & -0.046 & 0.664 & 0.087 \\
\hline 1.6 & 0.697 & 0.171 & 0.715 & 0.257 & 0.757 & 0.141 & 0.730 & 0.166 \\
\hline 1.8 & 0.784 & 0.153 & 0.759 & 0.240 & 0.807 & 0.160 & 0.746 & 0.312 \\
\hline 2.0 & 0.764 & 0.235 & 0.777 & 0.227 & 0.820 & 0.195 & 0.786 & 0.275 \\
\hline 2.2 & 0.720 & 0.346 & 0.696 & 0.456 & 0.754 & 0.356 & 0.709 & 0.477 \\
\hline
\end{tabular}

TABLE III. The best fit values for the parametric representation of $\mathrm{P}_{\mathrm{NWA}}$ in Eq. (20) for different values of $M_{B}$.

\begin{tabular}{|c|c|c|c|c|c|c|c|c|}
\hline \multirow[b]{2}{*}{$M_{T}(\mathrm{TeV})$} & \multicolumn{2}{|c|}{$W B W t$} & \multicolumn{2}{|c|}{$W B Z b$} & \multicolumn{2}{|c|}{$Z B W t$} & \multicolumn{2}{|c|}{$Z B Z b$} \\
\hline & $A_{1}$ & $A_{2}$ & $A_{1}$ & $A_{2}$ & $A_{1}$ & $A_{2}$ & $A_{1}$ & $A_{2}$ \\
\hline 1.0 & 0.506 & -0.333 & 0.506 & -0.280 & 0.548 & -0.250 & 0.409 & 0.133 \\
\hline 1.2 & 0.652 & -0.178 & 0.646 & -0.159 & 0.653 & -0.111 & 0.542 & 0.105 \\
\hline 1.4 & 0.741 & -0.046 & 0.721 & -0.015 & 0.727 & -0.009 & 0.625 & 0.166 \\
\hline 1.6 & 0.776 & 0.116 & 0.759 & 0.133 & 0.761 & 0.108 & 0.677 & 0.210 \\
\hline 1.8 & 0.807 & 0.195 & 0.796 & 0.189 & 0.760 & 0.222 & 0.673 & 0.351 \\
\hline 2.0 & 0.831 & 0.203 & 0.806 & 0.235 & 0.768 & 0.242 & 0.711 & 0.300 \\
\hline 2.2 & 0.781 & 0.345 & 0.757 & 0.346 & 0.720 & 0.362 & 0.648 & 0.442 \\
\hline
\end{tabular}

On the other hand, as can be seen in Fig. 4, $\mathrm{P}_{\mathrm{NWA}}$ becomes slightly higher than unity in the small but finite $\frac{\Gamma}{M}$ region before it starts to decrease. The differential crosssection distributions for WTHt processes of a $1.6 \mathrm{TeV}$ top partner for different choices of VLQ couplings are shown in Fig. 6. At very low decay widths, the finite width cross section of the WTHt processes is slightly smaller than predicted by the NWA because of a widening of the Breit-Wigner propagator, resulting in $\mathrm{P}_{\mathrm{NWA}}>1$. However, the VLQ energy distribution for a WTHt process receives an enhancement at lower energies for larger decay widths. This can cause the finite width cross section for WTHt processes to be higher than the NWA prediction, resulting in $\mathrm{P}_{\mathrm{NWA}}<1$.

The functional behavior of $\mathrm{P}_{\mathrm{NWA}}$ can be approximated by a piecewise function of the form in Eq. (21):

$$
\mathrm{P}_{\mathrm{NWA}, V Q H q}\left(\frac{\Gamma_{Q}}{M_{Q}}\right)=\left\{\begin{array}{ll}
1+A B \frac{\Gamma_{Q}}{M_{Q}}-\left(\frac{A B}{x_{0}}\right)\left(\frac{\Gamma_{Q}}{M_{Q}}\right)^{2}, & \frac{\Gamma_{Q}}{M_{Q}}<x_{0} \\
1-A\left(1-\exp \left(-B\left(\frac{\Gamma_{Q}}{M_{Q}}-x_{0}\right)\right)\right), & \frac{\Gamma_{Q}}{M_{Q}} \geq x_{0}
\end{array} .\right.
$$

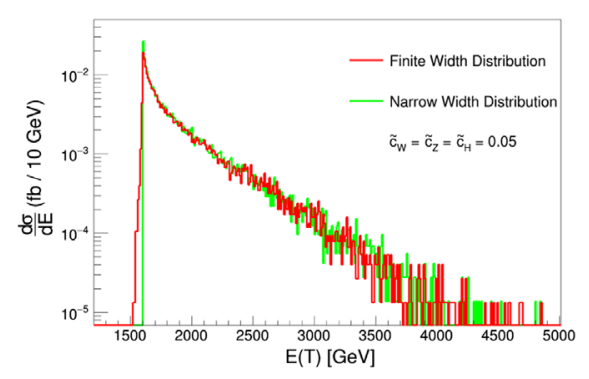

(a)

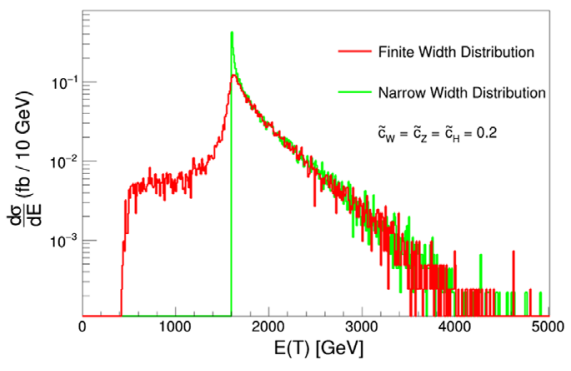

(b)

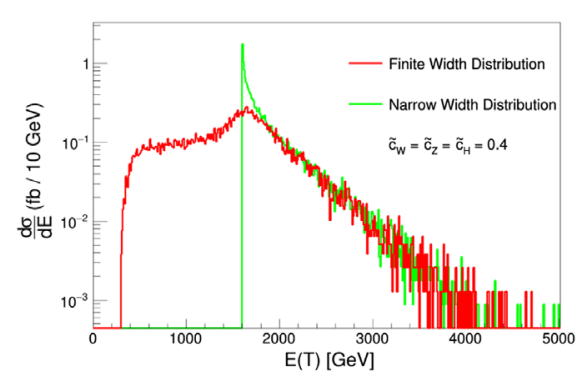

(c)

FIG. 6. Distribution of a differential cross section of singly produced $T$ of mass $M_{T}=1.6 \mathrm{TeV}$ at narrow width and finite width as obtained from a MadGraph_amC@NLO simulation of the WTHt process. The coupling values $\tilde{c}_{W}$ are set at $0.05,0.20$, and 0.40 , respectively in (a)-(c). All distributions assume that $\tilde{c}_{W}=\tilde{c}_{Z}=\tilde{c}_{H}$. 
TABLE IV. The best fit values for the parametric representation of $\mathrm{P}_{\mathrm{NWA}}$ in Eq. (21) for different values of $M_{T / B}$.

\begin{tabular}{|c|c|c|c|c|c|c|c|c|c|c|c|c|}
\hline \multirow[b]{2}{*}{$M_{T}(\mathrm{TeV})$} & \multicolumn{3}{|c|}{$W T H t$} & \multicolumn{3}{|c|}{$\mathrm{ZTHt}$} & \multicolumn{3}{|c|}{$W B H b$} & \multicolumn{3}{|c|}{$\mathrm{ZBHb}$} \\
\hline & A & $B$ & $x_{0}$ & $A$ & $B$ & $x_{0}$ & A & $B$ & $x_{0}$ & $A$ & $B$ & $x_{0}$ \\
\hline 1.0 & 0.057 & 35.032 & 0.052 & $\sim 0$ & $\sim 0$ & $\ldots^{\mathrm{a}}$ & 0.031 & 96.784 & 0.055 & 0.162 & 10.297 & 0.042 \\
\hline 1.2 & 0.189 & 10.005 & 0.035 & 0.078 & 20.616 & 0.047 & 0.141 & 11.510 & 0.042 & 0.296 & 7.985 & 0.031 \\
\hline 1.4 & 0.319 & 7.923 & 0.026 & 0.199 & 9.290 & 0.030 & 0.264 & 8.116 & 0.030 & 0.412 & 7.673 & 0.026 \\
\hline 1.6 & 0.433 & 7.801 & 0.022 & 0.321 & 7.936 & 0.026 & 0.379 & 7.633 & 0.025 & 0.514 & 7.990 & 0.022 \\
\hline 1.8 & 0.522 & 8.771 & 0.023 & 0.428 & 8.072 & 0.023 & 0.476 & 8.204 & 0.023 & 0.588 & 9.407 & 0.023 \\
\hline 2.0 & 0.613 & 9.329 & 0.021 & 0.526 & 8.456 & 0.021 & 0.568 & 8.773 & 0.023 & 0.668 & 10.275 & 0.023 \\
\hline 2.2 & 0.658 & 12.082 & 0.024 & 0.601 & 9.896 & 0.022 & 0.633 & 10.563 & 0.023 & 0.705 & 13.737 & 0.025 \\
\hline
\end{tabular}

${ }^{\mathrm{a}} \mathrm{P}_{\mathrm{NWA}, Z T H t} \approx 1.0$ for all $\frac{\Gamma}{M}$ at $M_{T}=1.0 \mathrm{TeV}$.

The two parts of the function are chosen such that $\mathrm{P}_{\mathrm{NWA}}\left(x_{0}\right)=1$ and that both functions and their derivatives are continuous at the joining point $x_{0}$. The values of the $A, B$, and $x_{0}$ parameters for different processes with top and bottom partners decaying to the Higgs boson, subject to the constraints $B>0$ and $0<x_{0} \leq 0.1$, are obtained by a least squared error fit to the observed values of the correction factor from simulation and tabulated in Table IV.

\section{REINTERPRETATION OF LIMITS FROM EXISTING ANALYSES}

The proposed interpretation strategy in Eq. (17) allows for a more comprehensive representation of the search results that are currently ongoing in the ATLAS and CMS experiments. To illustrate the flexibility this interpretation strategy offers, we take the ATLAS analysis in [30] and the CMS analysis in [33] as examples. Both analyses target the search for singly produced top partners $\left(T_{+\frac{2}{3}}\right)$ that eventually decay to a $Z$ boson, decaying into a pair of electrons or muons and a top quark. The ATLAS search focuses on two orthogonal analysis channels-the boosted dilepton channel and the trilepton channel. The boosted dilepton channel looks for a $Z$ boson decaying into a pair of electrons or muons as well as a boosted jet identified as the hadronic shower of the top quark. The trilepton channel includes an additional electron or muon from the leptonic decay of the $W$ boson, emerging from the decay of the top quark produced together with the $Z$ boson. This analysis performs a statistical combination of the two channels, and the ATLAS Collaboration reported the exclusion limit on the $W T Z t$ process cross section for masses in the range $0.7-2.0 \mathrm{TeV}$ and coupling values $\kappa$ between 0.1 and 1.6 following the parametrization prescribed in [39]. The aforementioned analysis has been stored as an entry in the HEPData [56] repository. The exclusion limits on the $W T Z t$ process cross section as a function of $M_{T}$ and $\kappa$ are available in this HEPData entry [57]. On the other hand, the CMS analysis introduces a ten-category search strategy based on a combination of lepton flavor from the $Z$ boson decay and a resolution of the $t$ quark decay products. In addition to calculating statistical limits on the $W T Z t$ process cross section under the NWA, assuming a coupling $c_{W}=0.5$ and $\mathrm{BR}(T \rightarrow W b)=0.5, \mathrm{BR}(T \rightarrow Z t)=$ $\mathrm{BR}(T \rightarrow H t)=0.25$ for $0.7 \mathrm{TeV} \leq M_{T} \leq 1.7 \mathrm{TeV}$, the CMS Collaboration also reported finite width crosssection limits for $\frac{\Gamma_{T}}{M_{T}}=10 \%, 20 \%$, and $30 \%$ and $0.8 \mathrm{TeV} \leq$ $M_{T} \leq 1.6 \mathrm{TeV}$. However, instead of probing the variation of the analysis results in the coupling space, they reported their exclusion limit $\sigma_{\lim }$ as a function of the relative decay width $\frac{\Gamma_{T}}{M_{T}}$ and the top partner mass $M_{T}$.

Both of these analyses reported their limits to be chirality agnostic, depending only on the effective coupling strength of the VLQ couplings and not their chiral structure. The smallest VLQ mass considered in these analyses is $700 \mathrm{GeV}$, which is large enough that the approximations made in Sec. III can be applied. For instance, the relative contribution in decay width $\Gamma(T \rightarrow Z t)$ by the interference term between the left and right chiral couplings is $6 \times$ $\frac{\tilde{c}_{L, Z} \tilde{c}_{R, Z}}{\tilde{c}_{L, Z}^{2}+\tilde{c}_{R, Z}^{2}} \times\left(\frac{m_{Z}}{M_{T}}\right)^{2} \frac{m_{t}}{M_{T}}[38]$, which evaluates to a maximum value of 0.013 at $M_{T}=700 \mathrm{GeV}$. This suggests that the assumptions made in Sec. III apply to both of the analyses reported in $[30,33]$, and we can apply the proposed semianalytical framework to reinterpret their results.

Figure 7(a) gives a generalized representation of the limits reported in the CMS analysis [33]. To ensure a simplified visualization, we set $\tilde{c}_{H}=\tilde{c}_{Z}$, which allows $\operatorname{BR}(T \rightarrow Z t) \approx$ $\operatorname{BR}(T \rightarrow H t)$ in the large mass limit. This assumption is well motivated in light of the Goldstone equivalence theorem [58]; in the large $M_{Q}$ limit, the longitudinal polarization dominates the Z-boson-mediated decay of the top partner, which is related to the Higgs mode by a hypercharge rotation, independent of the $S U(2)$ representation of the VLQs [37,59]. As a result, the $Z$ and $H$ boson decay vertices receive similar coupling strengths and the partial decay widths become similar, resulting in almost equal branching ratios independently of the group representation. An equivalent representation of the limits reported by the ATLAS analysis [30] is given in Figure 7(b).

However, it should be emphasized that the assumption of $\tilde{c}_{H}=\tilde{c}_{Z}$, albeit well motivated, is necessary only for the purpose of convenient representation. We can perform a 


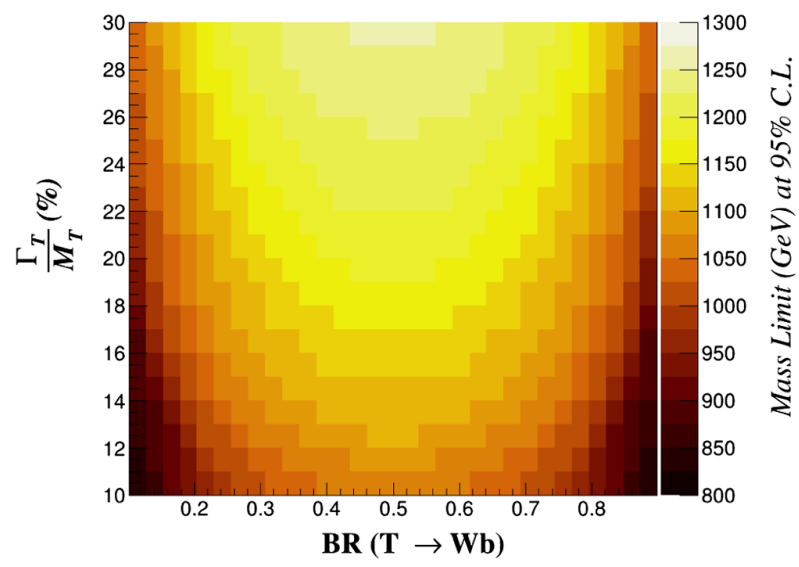

(a)

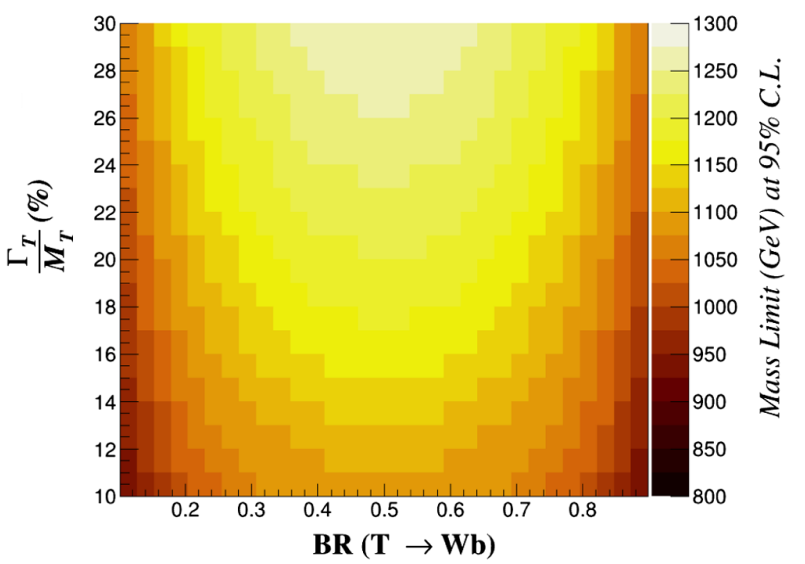

(b)

FIG. 7. Representation of the exclusion limits on VLQ mass in the $\frac{\Gamma_{T}}{M_{T}}-\mathrm{BR}(T \rightarrow W b)$ plane. This representation makes the assumption that $\tilde{c}_{Z}=\tilde{c}_{H}$, deeming the branching ratios in the $H$ and $Z$ channels equal in the large $M_{T}$ limit. The limits reported in the CMS analysis [33] are shown in (a) and the limits obtained in the ATLAS analysis [30] are represented in (b).

four-dimensional interpretation by allowing a generalized strategy of parametric reduction. We introduce the $f$ parameter,

$$
f=\frac{\tilde{c}_{H}}{\tilde{c}_{Z}},
$$

which defines a plane of projection in the four-dimensional hyperspace of (17). This parametrization expresses the branching ratios as a function of $\frac{\tilde{C}_{Z}}{\tilde{c}_{W}}$ in the large $M_{Q}$ limit:

$$
\begin{aligned}
\operatorname{BR}(T \rightarrow W b) & \approx \frac{1}{1+\left(1+f^{2}\right) \frac{\tilde{c}_{Z}^{2}}{\tilde{c}_{W}^{2}}}, \\
\operatorname{BR}(T \rightarrow Z t) & \approx \frac{1-\mathrm{BR}(T \rightarrow W b)}{1+f^{2}}, \\
\operatorname{BR}(T \rightarrow H t) & \approx f^{2} \times \mathrm{BR}(T \rightarrow Z t) .
\end{aligned}
$$

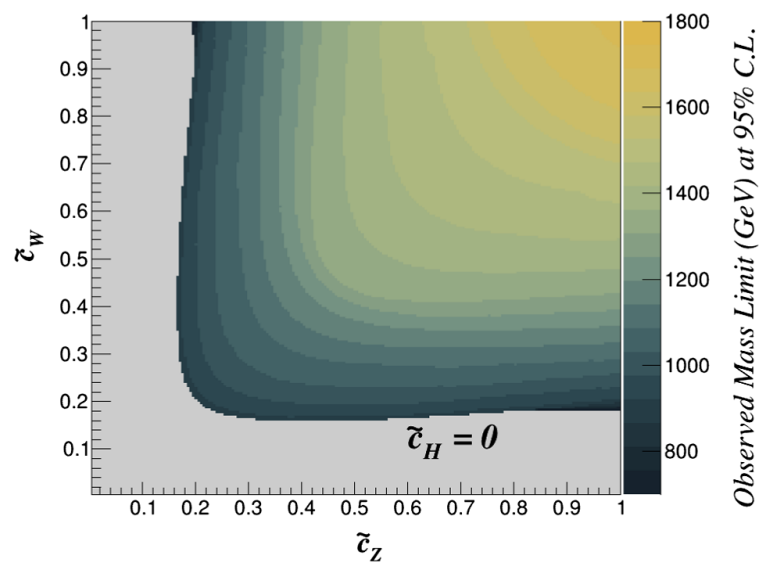

(a)
For a given choice of $f$, the contours for constant branching ratios are represented by vertical straight lines in the $\frac{\Gamma_{T}}{M_{T}}-\mathrm{BR}(T \rightarrow W b)$ plane. At the large $M_{Q}$ limit, the branching ratios often become independent of the VLQ mass as well as the couplings for certain group representations $[37,39]$. Hence, the $f$-factor-based reduction strategy makes it trivial to evaluate the sensitivity of an analysis in model-specific contexts.

Evidently, the representation of VLQ mass limits as a function of $c_{W}$ and $c_{Z}$ in the limit of $\operatorname{BR}(T \rightarrow Z t) \approx$ $\mathrm{BR}(T \rightarrow H t)$ proposed in [30] corresponds to the special case of $f=1$. However, the proposed framework in Eq. (17) can accommodate other choices of $f$ to probe the exclusion limits on alternate projections of the parametric hyperspace. We illustrate this in Fig. 8, where we numerically reinterpret the limits reported in the ATLAS analysis [30] for alternate choices of $f$.

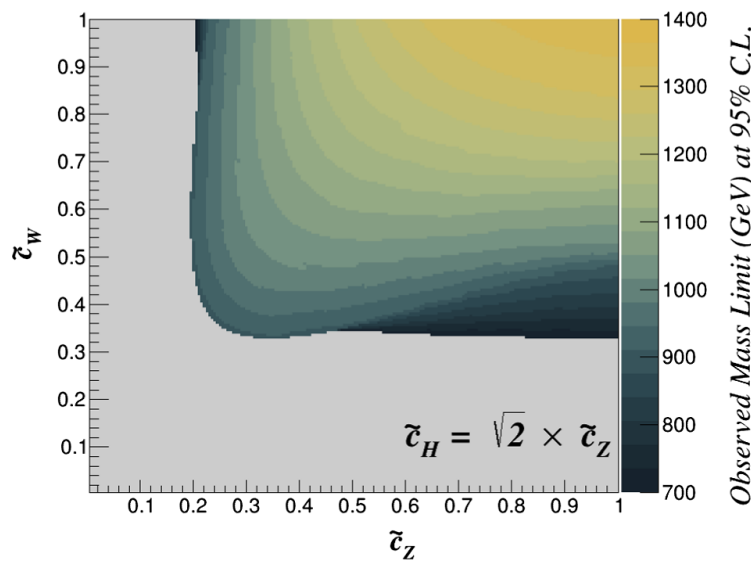

(b)

FIG. 8. Reinterpretation of observed limits on top partner mass from [30], plotted as a function of $\tilde{c}_{W}$ and $\tilde{c}_{Z}$ for (a) $f=0$ and (b) $f=\sqrt{2}$. The gray regions enclose a parametric space not covered within the sensitivity of the analysis. 
TABLE V. Comparison of reduced cross-section values calculated from Eq. (25) with the values reported in [33]. The values show good agreement, with the difference being at most $\mathcal{O}(10 \%)$ in most cases.

\begin{tabular}{lccc}
\hline \hline$M_{T}(\mathrm{TeV})$ & $\frac{\Gamma_{T}}{M_{T}}(\%)$ & $\hat{\sigma}(\mathrm{pb})$ from $(25)$ & $\hat{\sigma}(\mathrm{pb})$ from $[33]$ \\
\hline 1.0 & 10 & 192 & 183 \\
& 20 & 92 & 87 \\
& 30 & 59 & 55 \\
1.2 & 10 & 141 & 145 \\
& 20 & 67 & 68 \\
& 30 & 42 & 43 \\
1.4 & 10 & 107 & 112 \\
& 20 & 50 & 52 \\
& 30 & 31 & 33 \\
1.6 & 10 & 80 & 85 \\
& 20 & 37 & 39 \\
& 30 & 23 & 29 \\
\hline \hline
\end{tabular}

In Fig. 8(a), we choose $f=0$, which eventually implies that $\operatorname{BR}(T \rightarrow H t)=0$. On the other hand, $f=\sqrt{2} \Rightarrow$ $\mathrm{BR}(T \rightarrow H t) \approx 2 \times \mathrm{BR}(T \rightarrow Z t)$ is chosen for the reinterpretation in Fig. 8(b). For a given choice of $f$, the contours of constant branching fractions are given by straight lines passing through the origin in these plots. As expected, the analysis is sensitive to relatively lower top partner masses for higher values of $f$.

We now demonstrate how the proposed interpretation strategy can be used to correlate the different strategies used in the aforementioned analyses and compare their results. The CMS analysis [33] adapted the interpretation proposed by Carvalho et al. [60]. If a particular analysis is mostly sensitive to a certain decay channel of the VLQ and is relatively insensitive to other decay channels, the excluded cross section $\sigma_{\text {lim }}$ in Eq. (17) becomes a function of the total VLQ decay width and not the individual choices of the couplings. This is because the change in branching ratio for an alternate choice of couplings that produce the same decay width merely applies as a variation in normalization of the signal hypothesis and hence is not reflected in the calculation of the exclusion limits for the process cross section. Hence, the excluded cross sections themselves can be represented as a function of $M_{Q}$ and $\frac{\Gamma_{Q}}{M_{Q}}$. The exclusion region can be identified by comparing the exclusion limits with the process cross section, which, according to Carvalho et al. [60], can be expressed with factorized couplings as given by the following equation:

$$
\sigma_{V Q A q}\left(M_{Q}, \vec{c}\right)=C_{\text {prod }}^{2} C_{\mathrm{dec}}^{2} \times \hat{\sigma}_{V Q A q}\left(M_{Q}, \Gamma_{Q}\right),
$$

where $C_{\text {prod }}$ and $C_{\text {dec }}$ are the couplings associated with the production and decay vertices of the singly produced VLQs and $\hat{\sigma}$ represents a reduced cross section that depends only

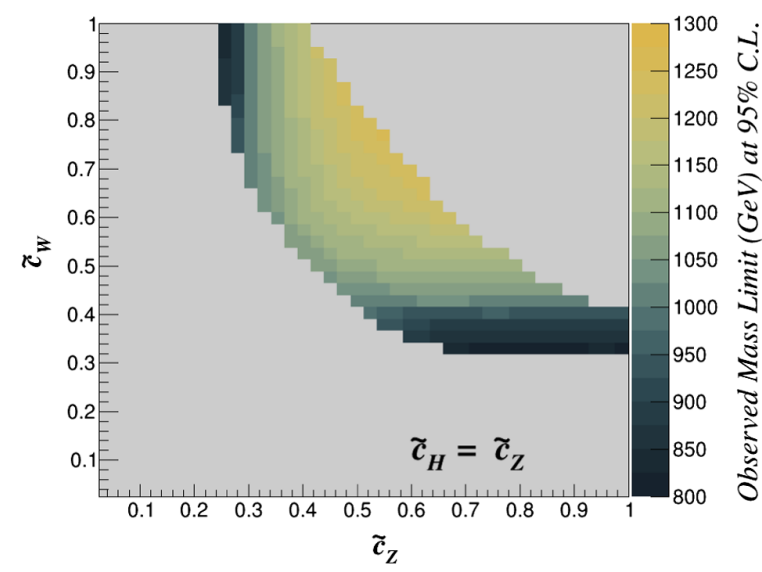

FIG. 9. Representation of the exclusion limits on VLQ mass from the CMS analysis [33] in the $\tilde{c}_{W}-\tilde{c}_{Z}$ plane. This representation makes an assumption $f=1$. The gray regions enclose a parametric space not covered within the sensitivity of the analysis.

on the choice of VLQ mass and the total decay width. Using the parametrization proposed in Secs. III and IV, we can express the so-called reduced cross section $\hat{\sigma}$ in terms of $\mathrm{P}_{\mathrm{NWA}}$ and $\sigma_{\mathrm{prod}, W T}^{\mathrm{NW}}$ for the $W T Z t$ process considered in the analyses by the equation

$\hat{\sigma}_{W T Z t}\left(M_{T}, \Gamma_{T}\right) \approx \frac{M_{T}^{2} \rho_{Z}(T)}{8 \pi g_{W}^{2} m_{Z}^{2}} \times \frac{\sigma_{\mathrm{prod}, W T}^{\mathrm{NW}}\left(M_{T}, \tilde{c}_{W}=1\right)}{\frac{\Gamma_{T}}{M_{T}}+A_{1} \frac{\Gamma_{T}^{2}}{M_{T}^{2}}+A_{2} \frac{\Gamma_{T}^{3}}{M_{T}^{3}}}$.

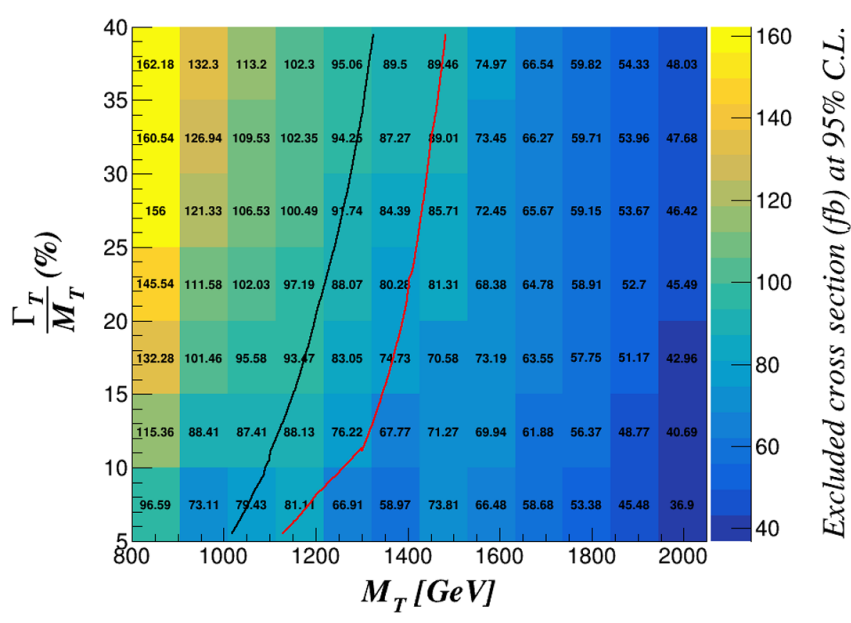

FIG. 10. Representation of exclusion limits from the ATLAS analysis [30] in the $\frac{\Gamma_{T}}{M_{T}}-M_{T}$ plane. The overlaid black line represents the exclusion limit for the branching fractions to the $W, Z$, and $H$ boson decay channels set at $0.5,0.25$, and 0.25 , respectively. The overlaid red line corresponds to the exclusion limit for branching fractions set atx $0.5,0.5$, and 0 , respectively. In both cases, the region to the left of the exclusion line is excluded. 
As shown in Table V, Eq. (25) can faithfully predict the reduced cross section that lies at the heart of the interpretation strategy proposed in [60]. This allows us to recast the limits reported in the CMS analysis [33] as exclusion limits on top partner mass as a function of the rescaled couplings (Figure 9).

As a final example of the flexibility that the proposed interpretation strategy offers, we recast the limits from the ATLAS analysis [30] as a function of the relative decay width $\frac{\Gamma_{T}}{M_{T}}$ and the top partner mass $M_{T}$ in Figure 10. In such representations, however, the excluded region in the parametric hyperspace depends on the choice of branching ratio.

\section{CONCLUSION}

We have presented a relatively model-independent approach for interpretation of single VLQ searches. This approach, under a minimal set of assumptions, allows a flexible representation of the results from a VLQ search effort and also provides an avenue of translating results presented in one approach to another. The proposed framework can bridge the gap between experimental searches and their phenomenological reinterpretations in the context of most well motivated VLQ physics models. The novelty of this approach lies in its analytic approach, which makes model-dependent reinterpretations of the search results computationally inexpensive. By numerically recasting the results from two independent analyses, we have established the flexibility that the proposed framework offers in obtaining nontrivial, information-dense yet easyto-interpret representations of such search results. This also harmonizes the representation of single VLQ search results and hence provides a platform for the combination of such analyses, an exciting avenue for future work.

\section{ACKNOWLEDGMENTS}

The work of A. R., N. N., and T. A. is supported by the U.S. Department of Energy, Office of High Energy Physics, under Grant No. DE-SC0007890. N. C. acknowledges the support by FCT-Portugal, through Project No. CERN/FISPAR/0024/2019.
[1] F. Del Aguila and M. J. Bowick, Nucl. Phys. B224, 107 (1983).

[2] D. B. Kaplan, H. Georgi, and S. Dimopoulos, Phys. Lett. 136B, 187 (1984).

[3] K. Agashe, R. Contino, and A. Pomarol, Nucl. Phys. B719, 165 (2005).

[4] R. Contino, L. Da Rold, and A. Pomarol, Phys. Rev. D 75, 055014 (2007).

[5] N. Arkani-Hamed, A. G. Cohen, E. Katz, and A. E. Nelson, J. High Energy Phys. 07 (2002) 034.

[6] Y. Hosotani, S. Noda, and K. Takenaga, Phys. Lett. B 607, 276 (2005).

[7] I. Antoniadis, K. Benakli, and M. Quirós, New J. Phys. 3, 20 (2001).

[8] T. Appelquist, H.-C. Cheng, and B. A. Dobrescu, Phys. Rev. D 64, 035002 (2001).

[9] F. Del Aguila, L. Ametller, G. L. Kane, and J. Vidal, Nucl. Phys. B334, 1 (1990).

[10] J. A. Aguilar-Saavedra, J. High Energy Phys. 11 (2009) 030.

[11] M. Chala, J. Juknevich, G. Perez, and J. Santiago, J. High Energy Phys. 01 (2015) 092.

[12] C. Bini, R. Contino, and N. Vignaroli, J. High Energy Phys. 01 (2012) 157.

[13] J. P. Araque, N. F. Castro, and J. Santiago, J. High Energy Phys. 11 (2015) 120.

[14] ATLAS Collaboration, J. High Energy Phys. 08 (2015) 105.

[15] ATLAS Collaboration, Phys. Rev. D 91, 112011 (2015).

[16] ATLAS Collaboration, J. High Energy Phys. 11 (2014) 104.

[17] CMS Collaboration, Phys. Rev. D 93, 012003 (2016).

[18] CMS Collaboration, Phys. Rev. D 93, 112009 (2016).
[19] CMS Collaboration, Phys. Rev. Lett. 112, 171801 (2014).

[20] ATLAS Collaboration, Eur. Phys. J. C 76, 442 (2016).

[21] ATLAS Collaboration, J. High Energy Phys. 07 (2018) 089.

[22] ATLAS Collaboration, J. High Energy Phys. 10 (2017) 141.

[23] ATLAS Collaboration, J. High Energy Phys. 08 (2017) 052.

[24] ATLAS Collaboration, J. High Energy Phys. 12 (2018) 039.

[25] ATLAS Collaboration, Phys. Rev. D 98, 092005 (2018).

[26] ATLAS Collaboration, Phys. Rev. Lett. 121, 211801 (2018).

[27] CMS Collaboration, J. High Energy Phys. 11 (2017) 085.

[28] CMS Collaboration, Phys. Lett. B 779, 82 (2018).

[29] CMS Collaboration, J. High Energy Phys. 08 (2018) 177.

[30] ATLAS Collaboration, Phys. Rev. D 98, 112010 (2018).

[31] ATLAS Collaboration, J. High Energy Phys. 05 (2019) 164.

[32] ATLAS Collaboration, J. High Energy Phys. 05 (2019) 041.

[33] CMS Collaboration, Phys. Lett. B 781, 574 (2018).

[34] CMS Collaboration, Eur. Phys. J. C 79, 90 (2019).

[35] CMS Collaboration, J. High Energy Phys. 06 (2018) 031.

[36] CMS Collaboration, J. High Energy Phys. 01 (2020) 036.

[37] J. A. Aguilar-Saavedra, R. Benbrik, S. Heinemeyer, and M. Pérez-Victoria, Phys. Rev. D 88, 094010 (2013).

[38] O. Matsedonskyi, G. Panico, and A. Wulzer, J. High Energy Phys. 12 (2014) 097.

[39] M. Buchkremer, G. Cacciapaglia, A. Deandrea, and L. Panizzi, Nucl. Phys. B876, 376 (2013).

[40] B. Fuks and H.-S. Shao, Eur. Phys. J. C 77, 135 (2017).

[41] A. Atre, G. Azuelos, M. Carena, T. Han, E. Ozcan, J. Santiago, and G. Unel, J. High Energy Phys. 08 (2011) 080.

[42] G. Cacciapaglia, A. Deandrea, D. Harada, and Y. Okada, J. High Energy Phys. 11 (2010) 159. 
[43] J. Alwall, R. Frederix, S. Frixione, V. Hirschi, F. Maltoni, O. Mattelaer, H.-S. Shao, T. Stelzer, P. Torrielli, and M. Zaro, J. High Energy Phys. 07 (2014) 079.

[44] J. Alwall, M. Herquet, F. Maltoni, O. Mattelaer, and T. Stelzer, J. High Energy Phys. 06 (2011) 128.

[45] N. Vignaroli, Phys. Rev. D 89, 095027 (2014).

[46] D. Greco and D. Liu, J. High Energy Phys. 12 (2014) 126.

[47] G. Cacciapaglia, T. Flacke, M. Park, and M. Zhang, Phys. Lett. B 798, 135015 (2019).

[48] B. A. Dobrescu and F. Yu, J. Phys. G 45, 08LT01 (2018).

[49] R. Benbrik, E. B. Kuutmann, D. B. Franzosi et al., J. High Energy Phys. 05 (2020) 028.

[50] M. J. Dolan, J. Hewett, M. Krämer, and T. Rizzo, J. High Energy Phys. 07 (2016) 039.

[51] M. Chala, Phys. Rev. D 96, 015028 (2017).
[52] J. A. Aguilar-Saavedra, D. E. López-Fogliani, and C. Muñoz, J. High Energy Phys. 06 (2017) 095.

[53] M. Tanabashi et al. (Particle Data Group), Phys. Rev. D 98, 030001 (2018).

[54] N. Kauer, Phys. Lett. B 649, 413 (2007).

[55] D. Berdine, N. Kauer, and D. Rainwater, Phys. Rev. Lett. 99, 111601 (2007).

[56] E. Maguire, L. Heinrich, and G. Watt, J. Phys. Conf. Ser. 898, 102006 (2017).

[57] ATLAS Collaboration, Phys. Rev. D 98, 112010 (2018), HEPData entry for ATLAS analysis [30].

[58] M. S. Chanowitz and M. K. Gaillard, Nucl. Phys. B261, 379 (1985).

[59] G. Panico and A. Wulzer, The Composite Nambu-Goldstone Higgs (Springer, New York, 2016).

[60] A. Carvalho, S. Moretti, D. O'Brien, L. Panizzi, and H. Prager, Phys. Rev. D 98, 015029 (2018). 